\title{
Advanced TEM characterization of new electrical contacts for high efficiency c-Si solar cells
}

\author{
Q. Jeangros ${ }^{1}$, J. Geissbühler ${ }^{1,2}$, J. Bullock ${ }^{3,4,5}$, A. Javey ${ }^{3,4}$, S. De Wolf ${ }^{1}$, A. Hessler-Wyser ${ }^{1}$, C. Ballif ${ }^{1,2}$ \\ 1. Photovoltaics and Thin Film Electronics Laboratory, Ecole Polytechnique Fédérale de Lausanne, \\ Neuchâtel, Switzerland \\ 2. PV center, Swiss Center for Electronics and Microtechnology, Neuchâtel, Switzerland \\ 3. Department of Electrical Engineering and Computer Sciences, University of California, Berkeley, \\ USA \\ 4. Materials Sciences Division, Lawrence Berkeley National Laboratory, Berkeley, USA. \\ 5. Research School of Engineering, The Australian National University, Canberra, Australia
}

High-efficiency crystalline silicon solar cells increasingly feature sophisticated electrical contacts that combine, often on the nanometer scale, numerous layers of specific chemistry and functionality. Precise diagnosis and control of the microstructure, crystallography and chemistry of each of these thin films present in the electrical contacts, along with their possible interactions during manufacturing, is essential to understand device limitations and maximize conversion efficiencies. In this presentation, we show through different examples how advanced transmission electron microscopy (TEM) techniques may assist in the development of new generations of high efficiency Si-based solar cells.

A first example deals with parasitic absorption losses at the front of Si heterojunction (SHJ) cells, which may be mitigated by replacing the thin p-type amorphous $\mathrm{Si}(\mathrm{a}-\mathrm{Si}: \mathrm{H})$ hole collector by a transparent metal oxide such as sub-stoichiometric molybdenum oxide $\left(\mathrm{MoO}_{\mathrm{x}}\right.$, with $\left.\mathrm{x}<3\right)$ [1]. While exhibiting high transparency and excellent hole collection properties, the replacement of doped a-Si: $\mathrm{H}_{\text {by }} \mathrm{MoO}_{\mathrm{x}}$ in SHJ cells poses several challenges in terms of device integration. Indeed, the full process must be optimized so that the $\mathrm{MoO}_{\mathrm{x}}$ layer is compatible with neighboring layers, without degradation during cell fabrication. In this context, TEM micrographs revealed the apparition of an interlayer between $\mathrm{MoO}_{\mathrm{x}}$ and the transparent conductive oxide (TCO) that may act as a carrier-blocking layer. This explained a noted decreased in device performance after contact-grid curing above $130{ }^{\circ} \mathrm{C}$, which is essential to evaporate solvents from the classical metallization paste and yield good electrical grid properties. To avoid such annealing step, we developed alternative low-temperature electrode deposition methods such as $\mathrm{Ni} / \mathrm{Cu}$ electroplating. However, direct electrodeposition of the electrode material onto the TCO layer may result in limited adhesion. Again, this finding was enabled by scanning (transmission) electron microscopy micrographs, coupled to energy-dispersive X-ray spectroscopy, identifying the presence of $\mathrm{nm}$-sized voids at this interface (Fig.1 [2]). The thermal evaporation of a thin $\mathrm{Cu}$ layer prior to electrodeposition could circumvent this issue to provide a good $\mathrm{Cu} / \mathrm{TCO}$ contact. Overall, these developments, assisted notably by electron microscopy, eventually lead to a $\mathrm{MoO}_{\mathrm{x}}$-based SHJ cell with a $\mathrm{Cu}$ electroplated grid demonstrating an efficiency of $22.5 \%$ with a fill factor over $80 \%$ [1].

Secondly, in a similar way, alkali metal fluoride compounds have gathered attention thanks to their electron-collection properties. Contacts based on these materials, combined with metal-oxide based hole collecting contacts, open the road towards impurity-doping free silicon photovoltaics [3]. These materials also appear as promising candidates for contacts to lowly doped n-type $\mathrm{Si}$ in partial rear contact cells [4]. But again, any integration of a new layer into the device requires a detailed assessment of its compatibility with the full manufacturing process, with TEM providing useful insight into nanometer scale structure and chemistry. 


\section{References:}

[1] J Geissbühler, J Werner, S.M.D Nicolas et al., Applied Physics Letters 104 (2015), p. 081601

[2] J Geissbühler, S. De Wolf, A. Faes et al., IEEE Journal of Photovoltaics 4 (2014), p. 1055

[3] J Bullock, M Hettick, J Geissbühler et al., Nature Energy 15031 (2016)

[4] J Bullock, P. Zheng, Q. Jeangros, et al.. Submitted (2016).

[5] Support is gratefully acknowledged from the Swiss National Science Foundation (project CRSII2 154474, "Impact of composition and nanometer scale DISorder in transparent Conductive Oxides: a new route to design materials with enhanced transport properties (DisCO)"), and the Department of Energy under the FPaceII project.
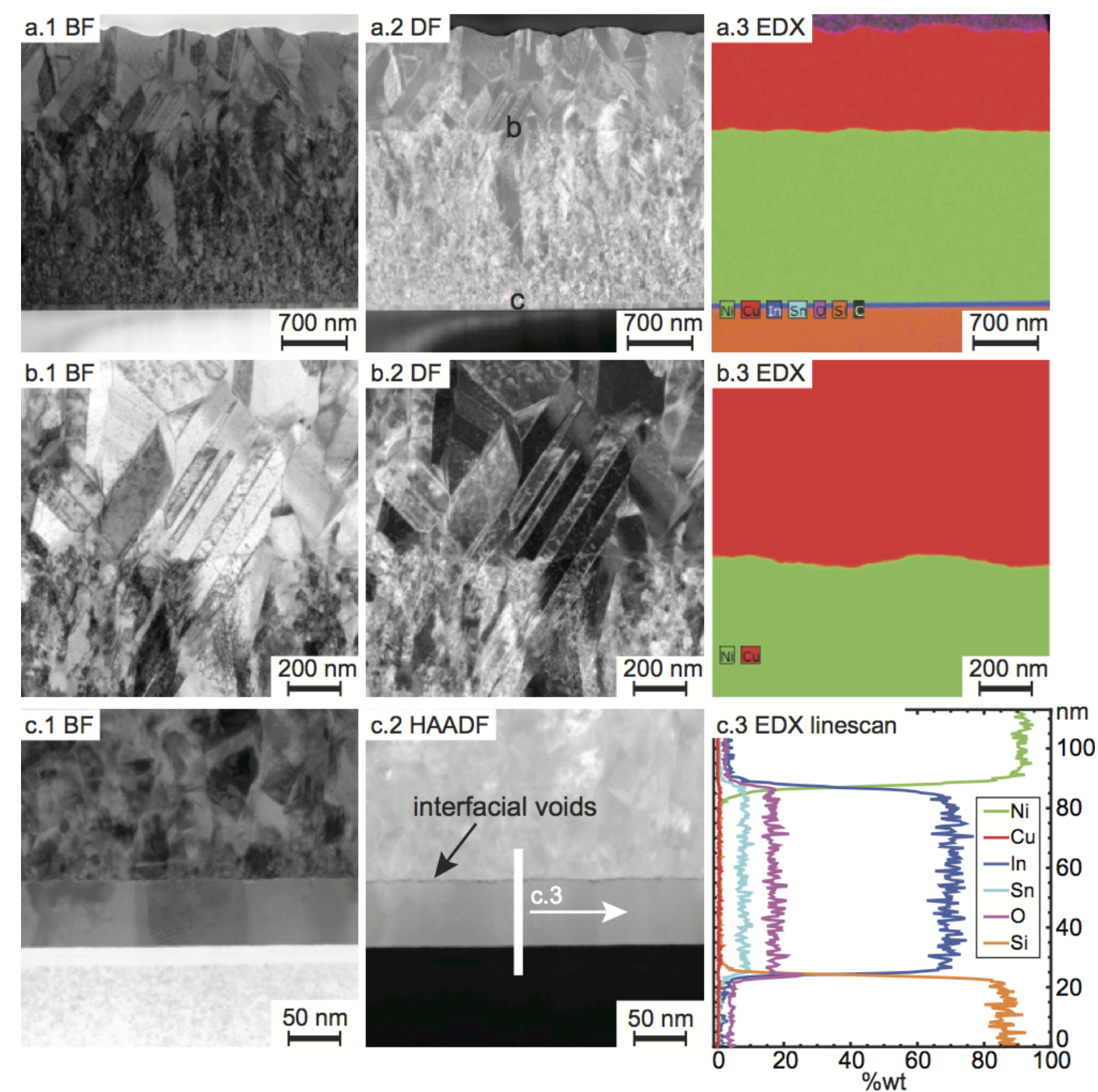

Figure 1. Focused ion beam-prepared cross section of the $\mathrm{Ni} / \mathrm{Cu}$ electroplated grid electrode, with a) scanning TEM (STEM) bight-field (BF) and dark-field (DF) images and corresponding energydispersive X-ray spectroscopy map of the full structure, b) STEM BF and DF images and EDX map of the $\mathrm{Ni} / \mathrm{Cu}$ interface, highlighting epitaxy between $\mathrm{Ni}$ and $\mathrm{Cu}$, and c) STEM BF and high-angle annular dark-field (HAADF) micrographs, with a corresponding linescan of the TCO (indium tin oxide) $/ \mathrm{Ni}$ interface. The arrow in c. 2 demonstrates the presence of interfacial voids between the TCO and Ni. 